\title{
The Analysis and Calculation of Artillery Breech Force Based on MATLAB
}

\author{
Zhaoqiang $\mathrm{Gu}^{1, \text { a }}$, Changchun $\mathrm{Di}^{1, \mathrm{~b}}$ \\ ${ }^{1}$ Department of Artillery Engineering, Ordnance Engineering College, Shijiazhuang 050003, China \\ a guxhhb@sina.com, bqiangfyyf@sina.com
}

Keywords: MATLAB; internal ballistic; artillery breech force; artillery;

\begin{abstract}
At present, the analysis and calculation of artillery breech force based on the clas sical interior ballistic theory describe the transformation law of interior ballistic parameters a nd optimize the related parameters to correct the error due to interior ballistic model proces sing, which leads to a larger error of recoil resistance change law and affects the accuracy of the recoil machine structure optimization design. Therefore, in this paper, the problem of artillery breech force analysis and calculation is solved by using numericalanalysis software MATLAB, with setting up solving model and the corresponding program. By comparison with the results of numerical simulation with the theoretical value, which indicates that the method has certain reliability and greatly improve the accuracy and efficiencyof the analysis and calculation of artillery breech force, with providing effective reference for further impr oving the accuracy of artillery recoil motion simulation.
\end{abstract}

\section{Classic artillery breech force calculation model}

In the artillery firing process, the powder of high pressure gas effect on body tube axial force called the artillery breech force ${ }^{[1]}$ is the main power of artillery recoil movement. By the knowledge of interior ballistics, the gunpowder gas effect time is about a few to 10 milliseconds, but its effect on artillery complicated situation.

In this paper, the projectile movement within artillery tube is analyzed. In this time period, the bottom of the chamber which is full of gunpowder gas and cone face are subjected to gas, with artillery barrels subjected to axial force component. The projectile moves with grooves turn guide side subjected to pressure and friction force. The sum of axial force component along artillery bore is known as the projectile forces acting on the grooves. By the above analysis, the artillery breech force is only associated with gunpowder inside the gas chamber pressure gradient distribution in this time period ${ }^{[2]}, \quad$ which is shown as in figure 1.

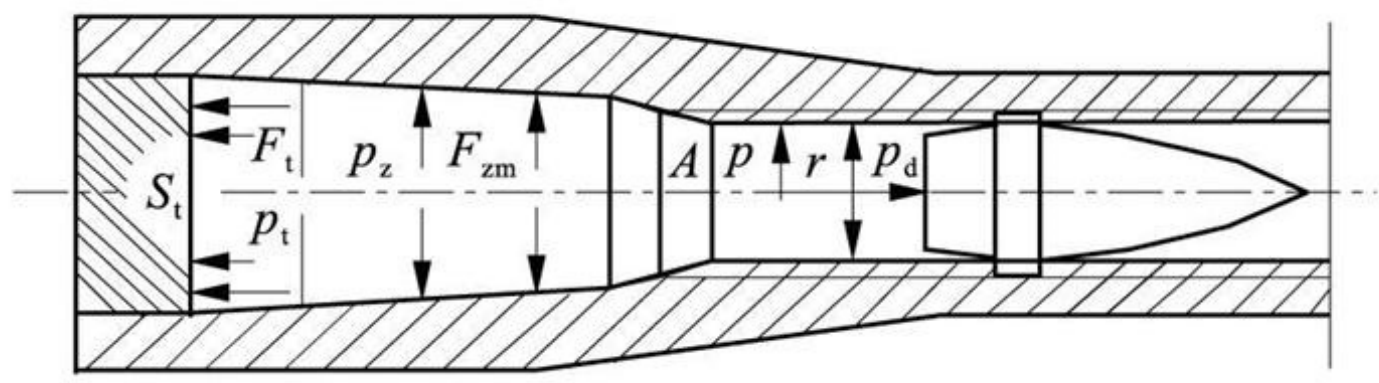

Fig. 1 Artillery breech force

The computational formula can be represented as:

$F_{p t}=F_{t}-F_{z m}-F_{d z}$

$F_{p t}$ - - the artillery breech force

$F_{t}{ }^{--}$the pressure of bottom of the chamber subjected to gas 
$F_{d z}{ }^{--}$the axial force component of grooves turn guide side subjected to projectile

$F_{z m}--$ the axial force component of chamber throat subjected to gas

\section{The pressure of bottom of the chamber subjected to gas}

Under the condition of basic hypothesis in classical interior ballistics theory, the breech pressure has following relationship with bore pressure.

$$
p_{t}=\frac{1+\frac{1}{2} \frac{\omega}{\varphi_{1} m}}{1+\frac{1}{3} \frac{\omega}{\varphi_{1} m}} p=\frac{\varphi_{1}+\frac{1}{2} \frac{\omega}{m}}{\varphi_{1}+\frac{1}{3} \frac{\omega}{m}} p \approx \frac{1}{\varphi}\left(\varphi_{1}+\frac{1}{2} \frac{\omega}{m}\right) p
$$

$\omega$--The quality of the charge

$m$--The quality of the projectile

$\varphi_{1}{ }^{--}$Calculating coefficient is of about 1.02 if only considering projectiles friction and rotation

$\varphi^{--}$calculated coefficient of the secondary power

$A_{t}-$-the bottom of the bore area

Usually, $\varphi=K+\frac{1}{3} \frac{\omega}{m} \approx \varphi_{1}+\frac{1}{3} \frac{\omega}{m}$

$$
F_{t}=p_{t} A_{t} \approx \frac{1}{\varphi}\left(\varphi_{1}+\frac{1}{2} \frac{\omega}{m}\right) p A_{t}
$$

\section{The axial force component of chamber throat subjected to gas}

As we known by the interior ballistics theory, gunpowder inside the gas chamber pressure gradient distribution is uneven. To calculate easily, $P_{z m}$ represents the gunpowder gas pressure on the cone of chamber, $A_{t}-A$ stands for the chamber cone perpendicular to the bore axis upward projection area. In a general way, there are almost no difference between $P_{z m}$ and $P_{\mathrm{t}}$

Therefore, $F_{z m}=\left(A_{t}-A\right) p_{t}$

The axial force component of grooves turn guide side subjected to projectile

The projectile motion equation is listed as follow.

$m \frac{d v}{d t}=A p_{d}-F_{d z}$

The relationship between $F_{d z}$ and $p_{d}$ is got.

$F_{d z}=\frac{1}{\varphi_{1}}\left(\varphi_{1}-1\right) A p_{d}$

From the formula (6), we can know that .

$p_{d}=\frac{\varphi_{1}}{\varphi} p$

Lastly, $F_{p t}$ has a linear relationship with $p$.

$F_{p t}=\frac{1}{\varphi}\left(1+\frac{1}{2} \frac{\omega}{m}\right) A p$

If the muzzle point pressure value is defined as $p_{g}$, the relationship of $F_{p t, g}$ and $p_{g}$ can be described as follow.

$F_{p t, g}=\frac{1}{\varphi}\left(1+\frac{1}{2} \frac{\omega}{m}\right) A p_{g}$

\section{Results}

The most commonly used means of differential equation is Runge-kutta method. MATLAB 
software integrates a variety of high efficiency, advanced solver, including ode23s, ode23t, ode15s for rigid continuous equation and ode23, ode45, ode113 for non rigid continuous equation, which are almost based on variable step solution and have the very good accuracy, stability and efficiency ${ }^{[3]}$.

Taking an artillery as an example, MATLAB calculation program has been set up according to the basic equation of artillery breech force calculating. Therefore, the artillery breech force and the projectile velocity transformation law with the time and schedule have been reached by means of main function call function for default solution based on ode45.
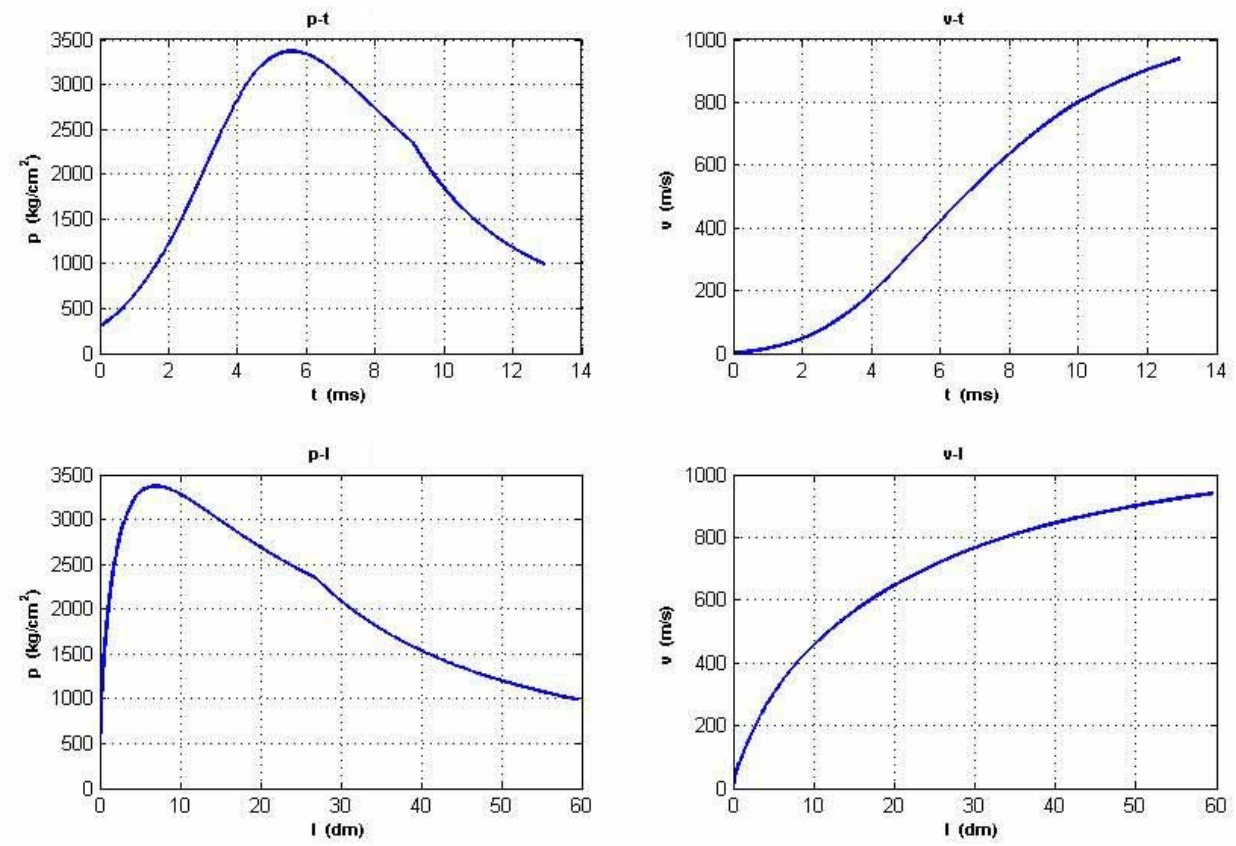

Fig. 2 The internal ballistic parameters simulation curve

In addition, the graphical analysis showed that breech pressure reaches maximum 336.59 $\mathrm{MPa}$ when time is $6.92 \mathrm{~ms}$. Meanwhile, artillery breech force achieves maximum 5488.40 $\mathrm{kN}$. The curves of pressure with stroke arranged in a parabolic approximation in the rise $\mathrm{p}$ hase and change more slowly in the decline phase, with short duration and high peak, dra matic changes ${ }^{[4]}$.

In the meantime, the projectile acceleration increases maximum and then decays with increasing time and speed with stroke shows a trend of gradual increase in. Within the cha mber pressure maximum points, the projectile of relative speed is $368.15 \mathrm{~m} / \mathrm{s}$, stroke $\mathrm{i}$ s $5.57 \mathrm{dm}$. The projectile relative speed reaches $940.43 \mathrm{~m} / \mathrm{s}$ in the muzzle points.

Since the type of artillery interior ballistic experiment data is difficult to obtain, this paper drew a comparison between the results of numerical simulation and the theoretical $\mathrm{V}$ alue provided from literature as shown in table 1. 
Table 1 The simulation results compared with the theoretical value

\begin{tabular}{|c|c|c|c|c|c|c|c|}
\hline \multirow{2}{*}{$\begin{array}{c}\text { Interior } \\
\text { ballistic } \\
\text { paramet } \\
\text { er }\end{array}$} & \multicolumn{3}{|c|}{ Internal pressure maximum points } & \multicolumn{3}{c|}{ The muzzle point } \\
\cline { 2 - 8 } & $l_{m} / d m$ & $p_{m} / M P a$ & $v_{m} /\left(m \cdot s^{-1}\right)$ & $t_{m} / m s$ & $p_{g} / M P a$ & $v_{g} /\left(m \cdot s^{-1}\right)$ & $t_{g} / m s$ \\
\hline $\begin{array}{c}\text { Value of } \\
\text { simulati } \\
\text { on }\end{array}$ & 6.92 & 336.59 & 368.15 & 5.57 & 98.83 & 940.43 & 12.94 \\
\hline $\begin{array}{c}\text { Theoreti } \\
\text { cal valu } \\
\text { e }\end{array}$ & 6.94 & 337 & 365 & 5.64 & 100.2 & 930 & 13.08 \\
\hline $\begin{array}{c}\text { Relative } \\
\text { error } \\
/ \%\end{array}$ & 0.29 & 0.15 & 0.86 & 1.24 & 1.36 & 1.12 & 1.07 \\
\hline
\end{tabular}

It is proved that the method of numerical simulation which the article adopts has good $\mathrm{p}$ recision and certain reliability due to that the internal ballistic parameters and the theore tical value relative error less than $2 \%$.

\section{Summary}

Through the in-depth analysis of artillery breech force calculation model based on the cl assical interior ballistic theory, four level explicit five order Runge Kutta method has been applied to numerical simulation, with considering algorithm accuracy and efficiency.

From the data processing results, it can be seen that the method has good accuracy and reliability, with providing effective reference for further enhancing artillery recoil motion si mulation accuracy.

\section{References}

[1] Xiangyan Zhang, Jianguo Zheng, Junrong Yang, Artillery design theory, Beijing Institute of Technology Press, (2005).

[2] Shuzi Gao, Yunsheng Chen, Yuelin Zhang, etc., Artillery recoil mechanism design, Weapons industry press, (1995).

[3] Zhenglin Wang, Ming Liu, Liangui Chen, MATLAB master, Electronic Industry Press, (2013).

[4] Lianrong Wang, Peiqin Zhang, The artillery interior ballistic calculation manual, National Defence Industry Press, (1987). 